\title{
New Deformation Twinning Mechanism Generates Zero Macroscopic Strain in Nanocrystalline Metals
}

\author{
X. L. Wu, ${ }^{1}$ X. Z. Liao, ${ }^{2}$ S. G. Srinivasan,,${ }^{3,4, *}$ F. Zhou, ${ }^{5}$ E. J. Lavernia, ${ }^{5}$ R. Z. Valiev, ${ }^{6}$ and Y. T. Zhu ${ }^{3,7, \dagger}$ \\ ${ }^{1}$ State Key Laboratory of Nonlinear Mechanics, Institute of Mechanics, Chinese Academy of Sciences, Beijing 100080, China \\ ${ }^{2}$ School of Aerospace, Mechanical \& Mechatronic Engineering, University of Sydney, NSW 2006, Australia \\ ${ }^{3}$ Materials Science and Technology Division, Los Alamos National Laboratory, Los Alamos, New Mexico 87544, USA \\ ${ }^{4}$ Department of Materials Science and Engineering, University of North Texas, Denton, Texas 76203, USA \\ ${ }^{5}$ University of California, Davis, California 95616, USA \\ ${ }^{6}$ Ufa State Aviation Technical University, 450000 Ufa, Russia \\ ${ }^{7}$ North Carolina State University, Raleigh, North Carolina 27695, USA
}

(Received 5 October 2007; published 5 March 2008)

\begin{abstract}
Macroscopic strain was hitherto considered a necessary corollary of deformation twinning in coarsegrained metals. Recently, twinning has been found to be a preeminent deformation mechanism in nanocrystalline face-centered-cubic (fcc) metals with medium-to-high stacking fault energies. Here we report a surprising discovery that the vast majority of deformation twins in nanocrystalline $\mathrm{Al}, \mathrm{Ni}$, and $\mathrm{Cu}$, contrary to popular belief, yield zero net macroscopic strain. We propose a new twinning mechanism, random activation of partials, to explain this unusual phenomenon. The random activation of partials mechanism appears to be the most plausible mechanism and may be unique to nanocrystalline fcc metals with implications for their deformation behavior and mechanical properties.
\end{abstract}

DOI: 10.1103/PhysRevLett.100.095701

When a metal is plastically deformed, its macroscopic strain is usually produced by deformation twinning and/or dislocation slip. Deformation twinning is a common and important phenomenon in materials including steels, shape-memory alloys, ferroelectric, and ferromagnetic materials, rocks, and geological minerals [1]. The twinning tendency of a face-centered-cubic (fcc) metal is largely determined by its stacking fault energy (SFE). For example, coarse-grained fcc metals with medium-to-high SFEs such as $\mathrm{Cu}, \mathrm{Al}$, and Ni usually prefer to deform by dislocation slip at room temperature and low strain rate, while low SFE fcc metals like Ag primarily deform by twinning [2].

Coarse-grained metals are believed to twin via conventional mechanisms including the pole [3], prismatic glide [4], faulted dipole [5], and others [6-8]. These deformation twins form by the glide of partials with the same Burgers vector on successive (111) planes. This collectively produces a net macroscopic strain at the grain level and changes the shape of the twinned grain as shown in Fig. 1(a).

Nanocrystalline (NC) fcc metals, in comparison, undergo deformation twinning via partials that are emitted from grain boundaries (GBs) and are believed to have the same Burgers vector [9-14]. We call this mechanism the monotonic activation of partials (MAP). Like conventional mechanisms in coarse-grained metals, the MAP twinning also generates a discernible shape change in the twinned grain. We indeed find evidence of MAP twins, albeit infrequently, in NC fcc metals [see Fig. 1(b)].

Twinning propensity dramatically increases in nanocrystalline grains of fcc metals with medium-to-high SFE [9-18]. Since both conventional and MAP twinning cause
PACS numbers: $81.30 . \mathrm{Kf}, 64.70 . \mathrm{K}-$ significant shape change and produce a net strain, it is widely assumed that enhanced twinning in nanocrystalline metals must also generate significant macroscopic strain compared to coarse-grained metals. However, in this Letter, we present experimental evidence that most of the deformation twins in nanocrystalline fcc metals produce zero macroscopic strain. We explain these unexpected findings by proposing a new twinning mechanism in nanocrystalline metals.

Results. - Three nanocrystalline fcc metals, $\mathrm{Al}, \mathrm{Ni}$, and $\mathrm{Cu}$, were used in this study. Their nanostructures were produced by cryogenic milling [11], cryotension [16], and high-pressure torsion [10]. Figure 2 shows typical high-resolution electron microscopy (HREM) images of deformation twins in nanocrystalline $\mathrm{Al}, \mathrm{Ni}$, and $\mathrm{Cu}$. As shown, GB segments are smooth even at locations where they intersect the twin boundaries. Thus, unlike the classi-
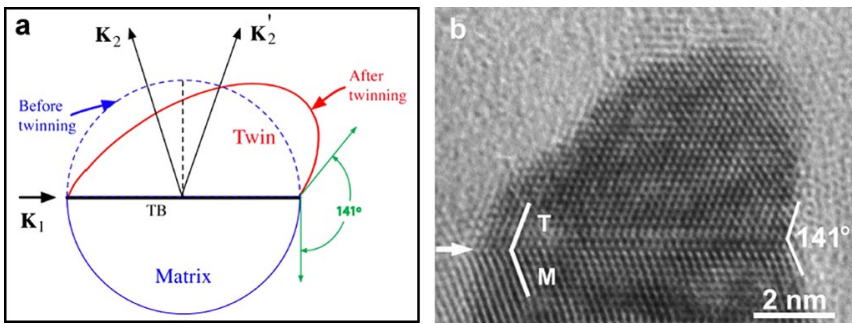

FIG. 1 (color online). (a) Conventional deformation twinning generates macroscopic strain and changes the grain shape. (b) High-resolution-electron micrograph of a MAP twin in nanocrystalline $\mathrm{Cu}$ synthesized by high-pressure torsion of 99.99 wt.\% purity coarse-grained copper disk, $0.5 \mathrm{~mm}$ thick and $10 \mathrm{~mm}$ diameter. Arrows indicate the twin boundaries. Twins and matrix are labeled $T$ and $M$, respectively. 

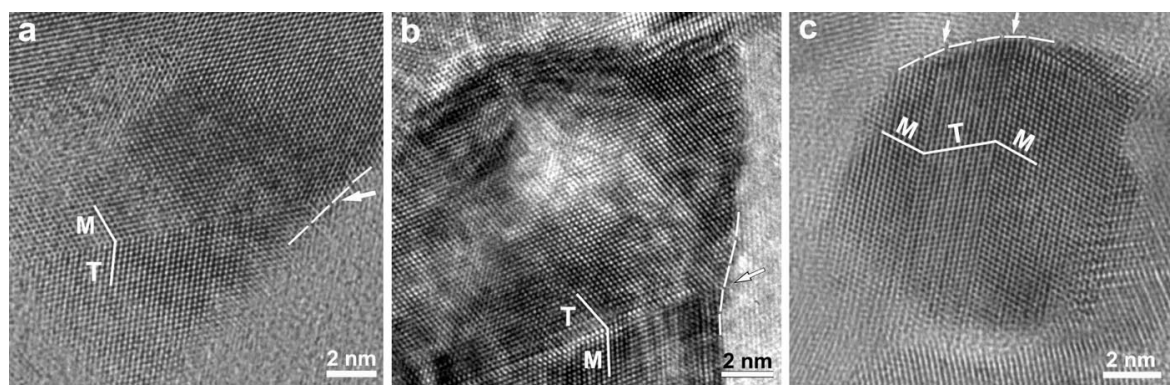

FIG. 2. HREM images of typical deformation twins in three nanocrystalline (NC) fcc metals. (a) Al, with 99.9 wt $\%$ purity and grain diameters between 0.5 to $1.0 \mu \mathrm{m}$, processed by cryomilling (i.e., by ball milling in liquid nitrogen for 8 hours); (b) electrodeposited Ni foils from Goodfellow, Inc. was immersed under liquid nitrogen and deformed by tension at a strain rate of $3 \times 10^{-3} \mathrm{~s}^{-1}$. The asreceived $150 \mu \mathrm{m}$ thick foils had an average grain size of $25 \mathrm{~nm}$. Preexisting growth twins were negligible in the $\sim 100$ grains we examined. (c) 99.99 wt.\% pure coarse-grained $\mathrm{Cu}$ disks, $0.5 \mathrm{~mm}$ thick and $10 \mathrm{~mm}$ diameter, were processed into NC-Cu via highpressure torsion (HPT) for 5 revolutions under $7 \mathrm{GPa}$ at room temperature and a very low strain rate of about $10^{-2} \mathrm{~s}^{-1}$. HPT produces fully dense NC materials without introducing measurable impurity into deformed samples. It is evident that the deformation twins did not change the shape of the grains. Arrows indicate the twin boundaries. The broken curves indicate GBs. Twins and matrix are labeled $T$ and $M$, respectively.

cal picture of deformation twinning illustrated in Fig. 1, the deformation twins shown in Fig. 2 did not change the morphology of the grains. This is a characteristic signature of zero macroscopic strain.

New twinning mechanism. - We propose that the twins that generate zero macrostrain were formed via a new twinning mechanism that we call random activation of partials (RAP), as explained below. Figure 3 shows the characteristic $A B C A B C A B C A B C A$ stacking in the fcc crystal, and $\mathbf{b}_{1}, \mathbf{b}_{2}$, and $\mathbf{b}_{3}$ are the three possible Shockley partials on a (111) plane. Although the microstain directions by these partials at the atomistic level are different, their glide produce the same atomic arrangement in the slip layer and layers above it, i.e., $A \rightarrow B, B \rightarrow C$, and $C \rightarrow A$. Since $\mathbf{b}_{1}+\mathbf{b}_{2}+\mathbf{b}_{3}=\mathbf{0}$, a random slip of equal numbers of these partials on successive layers of a (111) plane, as shown in Fig. 3(b), will produce a deformation twin with zero net macrostrain. It is important to realize that slip of first partials will impose a shape change to the grain and will increase the system strain energy. This, in turn, should drive the nucleation and propagation of compensating partials to lower system strain energy and eliminate the shape change of the grain. Slip of partials on successive $\{111\}$ planes is energetically more favorable than slip on separate planes [12].

To illustrate RAP, let us assume six partials are emitted randomly in the time sequence of $\mathbf{b}_{2}, \mathbf{b}_{3}, \mathbf{b}_{3}, \mathbf{b}_{1}, \mathbf{b}_{2}$, and $\mathbf{b}_{1}$ on atomic layer I, II, III, IV, V, and VI, respectively. Note that any other combination of these three partials in equal proportion would also work. The $\mathbf{b}_{\mathbf{2}}$ slip on layer I leads to a new stacking sequence of $A B C A C A B C A B C A B$ [see column "Step 1" in Fig. 3(b)]. Following the remaining sequence of steps shown in Fig. 3(b), we end up with the stacking sequence of $A B C A C B A C B A B C A$ [see column "Step 6" in Fig. 3(b)], creating a 6 layer twin $C B A C B A$. The two twin boundaries are marked as TB. Note that since the participating Burgers vectors sum to zero, RAP twin- ning must produce zero net macroscopic strain. Christian postulated an analogous mechanism to explain the fcc to hcp transformation in metals [19]. However, that transformation is driven by the Gibbs free energy difference unlike the local shear stresses in RAP. Mahajan et al. [20] proposed a formation mechanism for annealing twins that has some similarity with the RAP mechanism. The difference is that the former is associated with grain growth and grain boundary migration.

Grain boundaries are sources of Shockley partials and emit them randomly both in their locations and Burgers vector orientations. When a partial dislocation, say, $\mathbf{b}_{\mathbf{1}}$, slips across a grain and reaches GBs, some segments may become purely screw in nature. This screw partial can easily cross slip into the next plane, where it can slip by itself or transform into $\mathbf{b}_{2}$ or $\mathbf{b}_{3}$. Other GB sources of partials include dissociated dislocations on the GB [17], GB ledges [21], and/or triple junctions [15,22].

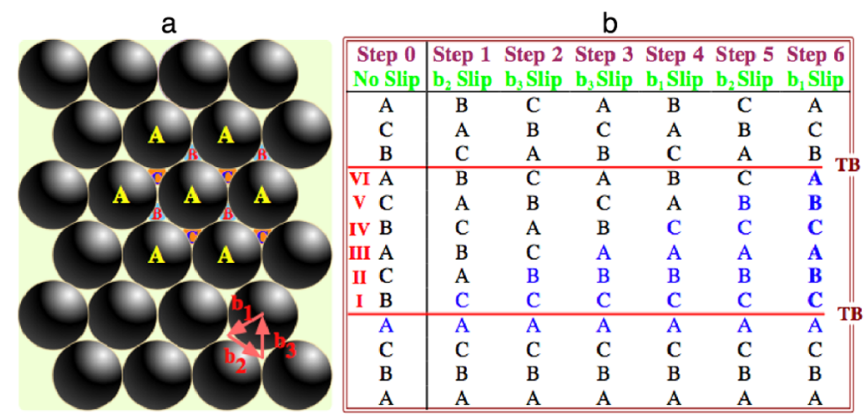

FIG. 3 (color online). The RAP twinning mechanism. (a) $A, B$, and $C$ are atom stacking sequences, and $\mathbf{b}_{1}, \mathbf{b}_{2}$, and $\mathbf{b}_{3}$ are the Burgers vectors of the partials. (b) Evolution of stacking sequence and formation of a RAP twin via the slip of partials $\mathbf{b}_{2}$, $\mathbf{b}_{3}, \mathbf{b}_{3}, \mathbf{b}_{1}, \mathbf{b}_{2}$, and $\mathbf{b}_{1}$ on layers I through VI, respectively. TB marks the two twin boundaries. Partials with negative Burgers vectors are not possible because they place two atoms on top of each other, increasing the system energy. 
For example, Fig. 4 illustrates the activation of a RAP process from a grain boundary junction through the emission of a Shockley partial dislocation loop, with the Burgers vector $\mathbf{b}_{\mathbf{1}}=\frac{\mathbf{a}}{\mathbf{6}}\left[\begin{array}{lll}\overline{\mathbf{1}} & \mathbf{1} & \mathbf{2}\end{array}\right]$ on a (111) plane. This dislocation loop is emitted from a GB junction and deposited on other GBs of a hexagonal grain. Part of the dislocation line segments parallel to grain edges $A B$ and $D E$ has pure screw character and can easily move in the GB to the next slip plane. On the next slip plane, $\mathbf{b}_{\mathbf{1}}$ can slip by itself under appropriate stress or produce other dislocations via the

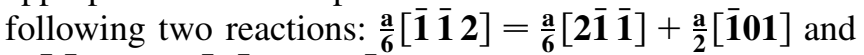
$\frac{\mathrm{a}}{\mathbf{6}}[\overline{\mathbf{1}} \overline{\mathbf{1}} \mathbf{2}]=\frac{\mathrm{a}}{\mathbf{6}}[\overline{\mathbf{1}} \mathbf{1} \overline{\mathbf{1}}]+\frac{\mathrm{a}}{2}[\mathbf{0} \overline{\mathbf{1}} \mathbf{1}]$. Thus it is possible to randomly emit the three Shockley partials on the next (111) slip plane.

Discussion. - A global shear stress cannot drive the three partials simultaneously. So what drives the $\mathbf{b}_{\mathbf{1}}, \mathbf{b}_{\mathbf{2}}$, and $\mathbf{b}_{\mathbf{3}}$ partial slips? We argue that it is possible for these partials to randomly nucleate and slip one at a time, driven by changing local shear stresses. These stresses could significantly differ from the global shear stress. That such a scenario is possible is revealed by atomistic simulations of stress concentration of 3-3.5 GPa, which is many times higher than the applied shear stress, near the edge of a stacking fault [23]. Furthermore, GB sliding and grain rotation, significant in nanocrystalline materials [24,25], alter local stress state [22] and/or changing the orientation of the twinning grain. These local stress variations can promote the random emission of partials. Other locations for high local stress concentration include triple junctions and GB steps. Also, once a twin is nucleated, it is relatively easy to grow the twin [12,13], thus explaining why the RAP twins should form instead of many stacking faults.

A global strain is widely believed to be necessary to relieve the global stress. Although the grains with RAP twins do not change their shape, they indeed contribute to the global strain by GB sliding and grain rotation. Also, during the RAP twinning, the applied stress, and the attendant accumulated energy, is effectively dissipated locally by the random nucleation and slip of three Shockley partials from GBs. However, their Burgers vectors add up to

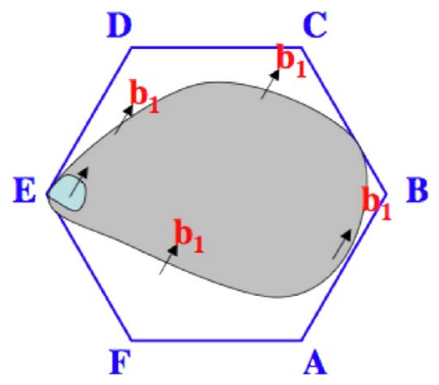

FIG. 4 (color online). Illustrating a possible RAP process. A Shockley dislocation loop with Burgers vector $\mathbf{b}_{\mathbf{1}}$ emitted from a GB junction $E$ (shaded blue) grows (shaded gray) and is deposited on other GBs of a hexagonal grain. Part of the dislocation line segments parallel to grain edges $A B$ and $D E$ has pure screw character and can move in the GB to the next slip plane. zero macroscopic strain, without violating laws of thermodynamics.

We hypothesize that RAP twinning ought to be favored over the MAP twinning because it imposes the least amount of strain accommodation on the neighbors of a twinning grain. On the contrary, the shape change produced in a MAP twin would generate significant stresses in the neighboring grains, forcing them to deform even when they are not favorably oriented to deform. Thus, the MAP twinning would require higher activation stresses. This analysis suggests that at higher applied stresses, MAP deformation twinning tendency increases, leading to a corresponding decrease in the percentage of RAP twins.

The above hypothesis is validated by our experiments in electrodeposited nanocrystalline $\mathrm{Ni}$ deformed under tension, rolling, and Hopkinson-bar impaction at liquid nitrogen temperature (LNT), with the stress increasing from tension to rolling to Hopkinson-bar impaction. As shown in Fig. 5, the percentage of the MAP twins increases as we go from tension to rolling and to Hopkinson-bar impact experiments. The RAP twins require relatively lower activation stresses and therefore remain predominant under all experimental conditions.

Alternately, it can be argued that the smooth GBs observed in Fig. 2 could be produced by first forming a twin in a large grain and then breaking that grain into NC grains. However, as explained below, this scenario is impossible in

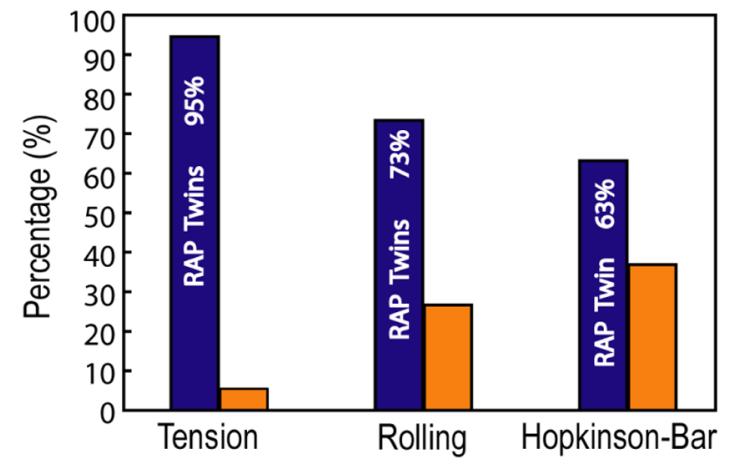

FIG. 5 (color online). Statistics were obtained from 18 to 38 twinned grains. Percentages of RAP and MAP (orange colored bars) deformation twins, as determined by HREM. The samples used were (i) electrodeposited nanocrystalline Ni deformed at LNT under tension at a strain rate of $3 \times 10^{-3} \mathrm{~s}^{-1}$ (to a strain of $4 \%$ and a corresponding maximum stress of about $1500 \mathrm{MPa}$ ), (ii) by cold rolling to a thickness reduction of $30 \%$ and (iii) under Hopkinson-bar impaction at LNT wherein $10 \mathrm{~mm}$ diameter specimens were placed between $10 \mathrm{~mm}$ diameter and $2500 \mathrm{~mm}$ long input and output bars. A $3 \mathrm{~kg}$ projectile, accelerated to a speed of $\sim 30 \mathrm{~m} \mathrm{~s}$, impacts the input bar generating an elastic compressive wave through the input bar. A part of this wave is reflected while the remainder transmits through the specimen, deforming it plastically. The strain rate was about $2.6 \times 10^{+3} \mathrm{~s}^{-1}$. The flow stress is estimated to be above $2.2 \mathrm{GPa}$. The changes of grain size distribution and mean grain sizes before and after three tests are negligible. The specimens did not fracture after cryogenic rolling or impaction. 
$\mathrm{NC} \mathrm{Ni}$, and very unlikely in the nanocrystalline $\mathrm{Al}$ and $\mathrm{Cu}$. The NC Ni had no observable change in grain size distribution after its deformation, indicating that no grain breakage happened. In $\mathrm{NC} \mathrm{Al}$ and $\mathrm{Cu}$, it was found that deformation twins do not form in grains larger than a certain critical size (e.g., $50 \mathrm{~nm}$ in $\mathrm{Cu}$ ), suggesting that the deformation twins were formed after the $\mathrm{NC}$ grains were formed first. Therefore, we are confident that the deformation twins shown in Fig. 2 were indeed formed by our RAP mechanism.

The discovery of RAP twinning has a profound impact on our understanding of the mechanical properties of NC fcc metals. First, contrary to popular belief, the twinning has negligible direct contribution to the macroscopic deformation at the grain level and consequently bulk strain at the sample level. Therefore, the bulk deformation in nanocrystalline fcc metals must be largely originated from the slip of full dislocations [16], GB sliding [24], grain rotation [24-26], and other deformation processes. Second, the RAP mechanism indirectly contributes to the bulk deformation by favorably reorienting the crystal lattice to change the applied resolved shear stresses on slip systems.

The predominance of RAP twinning in nanocrystalline fcc metals raises several fundamental questions. First, do similar twinning processes occur in nanocrystalline metals with other crystal structures such as bcc and hep? Second, what is the effect of RAP twinning on the mechanical behavior of nanocrystalline metals, especially their strain hardening rate and strain rate sensitivity? Third, does stacking fault energy influence the tendency of RAP twinning? Fourth, how does the RAP twinning affect the design of nanocrystalline alloys for superior mechanical properties? These issues need to be resolved using a combined modeling-experimental approach.

Summary. - We have discovered a significant new deformation twinning mechanism, random activation of partials or RAP, in NC fcc metals. The RAP mechanism does not produce macroscopic strain and is preferred because it requires relatively lower activation stresses. This new insight should have widespread implication because it must be factored in our understanding of the deformation behavior of NC metals, especially their strain hardening rate and strain rate sensitivity.

We are indebted to Professor J.P. Hirth, Professor Ke $\mathrm{Lu}$, Professor S. Mahajan, and Professor Terrence Mitchell for their suggestions and criticisms. The NSFC (No. 10721202, No. 50571110; X.L.W.), MOST (No. 2004CB619305; X.L. W.), CAS (No. KJCX2-YWM04; X. L. W.), Australian Research Council for financial support (Project ID: No. DP0772880; X.Z.L.), DOE Office of Science (S.G.S.), Office of Basic Energy Sciences (S. G. S.), IPP Program Office (Y.T.Z.), and Office of Naval Research (No. N00014-04-1-0370; E. J. L.) funded this work.
*Corresponding author.

Srivilliputhur@egw.unt.edu

Corresponding author.

ytzhu@ncsu.edu

[1] J. W. Christian and S. Mahajan, Prog. Mater. Sci. 39, 1 (1995).

[2] F. J. Humphreys and M. Hatherly, Recrystallization and Related Annealing Phenomena (Pergamon, New York, 1995), 3rd ed.

[3] A. Ookawa, J. Phys. Soc. Jpn. 25, 825 (1957).

[4] J. A. Venables, Philos. Mag. A 6, 379 (1961).

[5] M. Niewczas and G. Saada, Philos. Mag. A 82, 161 (2002).

[6] S. Mahajan and G. Y. Chin, Acta Metall. 21, 1353 (1973).

[7] S. Mahajan, M. L. Green, and D. Brasen, Metall. Trans. A 8, 283 (1977).

[8] N. Thompson, Proc. Phys. Soc. London Sect. B 66, 481 (1953).

[9] M. W. Chen, E. Ma, K. J. Hemker, H. W. Sheng, Y. M. Wang, and X. M. Cheng, Science 300, 1275 (2003).

[10] X.Z. Liao, Y.H. Zhao, S.G. Srinivasan, Y.T. Zhu, R.Z. Valiev, and D. Gunderov, Appl. Phys. Lett. 84, 592 (2004).

[11] X. Z. Liao, F. Zhou, E. J. Lavernia, S. G. Srinivasan, M. I. Baskes, D. W. He, and Y. T. Zhu, Appl. Phys. Lett. 83, 632 (2003).

[12] Y. T. Zhu, X.Z. Liao, S. G. Srinivasan, Y. H. Zhao, M. I. Baskes, F. Zhou, and E. J. Lavernia, Appl. Phys. Lett. 85, 5049 (2004).

[13] A. G. Frøseth, P. M. Derlet, and H. V. Swygenhoven, Adv. Eng. Mater. 7, 16 (2005).

[14] V. Yamakov, D. Wolf, S. R. Phillpot, A. K. Mukherjee, and H. Gleiter, Nat. Mater. 1, 45 (2002).

[15] H. V. Swygenhoven, P. M. Derlet, and A. G. Frøseth, Acta Mater. 54, 1975 (2006).

[16] X. L. Wu and E. Ma, Appl. Phys. Lett. 88, 231911 (2006).

[17] X. L. Wu and Y. T. Zhu, Appl. Phys. Lett. 89, 031922 (2006).

[18] D. Wolf, V. Yamakov, S. R. Phillpot, A. K. Mukherjee, and H. Gleiter, Acta Mater. 53, 1 (2005).

[19] J. W. Christian, The Theory of Transformations in Metals and Alloys (Pergamon, New York, 1965), 1st ed.

[20] S. Mahajan, C.S. Pande, M. A. Imam, and B. B. Rath, Acta Mater. 45, 2633 (1997).

[21] J. C. M. Li, Trans. Metall. Soc. AIME 227, 239 (1963).

[22] M. A. Meyers, A. Mishra, and D. J. Bensen, Prog. Mater. Sci. 51, 427 (2006).

[23] J. Wang and H.C. Huang, Appl. Phys. Lett. 85, 5983 (2004).

[24] N. Q. Chinh, P. Szommer, Z. Horita, and T. G. Langdon, Adv. Mater. 18, 34 (2006).

[25] X.Z. Liao, A. R. Kilmametov, R.Z. Valiev, H.S. Gao, X. D. Li, A. K. Mukherjee, J.F. Bingert, and Y. T. Zhu, Appl. Phys. Lett. 88, 021909 (2006).

[26] Z.W. Shan, E. A. Stach, J.M. K. Wiezorek, J. A. Knapp, D. M. Follstaedt, and S.X. Mao, Science 305, 654 (2004). 